\title{
When Does Channel-Output Feedback Enlarge the Capacity Region of the Two-User Linear Deterministic Interference Channel?
}

\author{
Victor Quintero $^{1}$, Samir M. Perlaza ${ }^{1}$, Iñaki Esnaola ${ }^{2}$, and Jean-Marie Gorce $^{1}$ ^ \\ ${ }^{1}$ Institut National de Recherche en Informatique et en Automatique (INRIA), \\ CITI Laboratory, 6 Av. des Arts, 69621, Villeurbanne, France. \\ 2 The University of Sheffield, Dep. of Automatic Control and Systems Engineering, \\ Mappin Street, Sheffield, S1 3JD, United Kingdom. \\ \{victor.quintero-florez, samir.perlaza\}@inria.fr, esnaola@sheffield.ac.uk, \\ jean-marie.gorce@inria.fr
}

\begin{abstract}
The two-user linear deterministic interference channel (LDIC) with noisy channel-output feedback is fully described by six parameters that correspond to the number of bit-pipes between each transmitter and its corresponding intended receiver, i.e., $\vec{n}_{11}$ and $\vec{n}_{22}$; between each transmitter and its corresponding non-intended receiver i.e., $n_{12}$ and $n_{21} ;$ and between each receiver and its corresponding transmitter, i.e., $\overleftarrow{n}_{11}$ and $\overleftarrow{n}_{22}$. An LD-IC without feedback corresponds to the case in which $\overleftarrow{n}_{11}=\overleftarrow{n}_{22}=0$. The capacity region of the linear deterministic interference channel with noisy channeloutput feedback is denoted by $\mathcal{C}\left(\vec{n}_{11}, \vec{n}_{22}, n_{12}, n_{21}, \overleftarrow{n}_{11}, \overleftarrow{n}_{22}\right)$. This paper presents the exact conditions on $\overleftarrow{n}_{11}$ (resp. $\overleftarrow{n}_{22}$ ) for observing an improvement in the capacity region $\mathcal{C}\left(\vec{n}_{11}, \vec{n}_{22}, n_{12}, n_{21}, \overleftarrow{n}_{11}, 0\right)$ (resp. $\left.\mathcal{C}\left(\vec{n}_{11}, \vec{n}_{22}, n_{12}, n_{21}, 0, \overleftarrow{n}_{22}\right)\right)$ when compared to $\mathcal{C}\left(\vec{n}_{11}, \vec{n}_{22}, n_{12}, n_{21}\right.$ $0,0)$, for any 4 -tuple $\left(\vec{n}_{11}, \vec{n}_{22}, n_{12}, n_{21}\right) \in \mathbb{N}^{4}$. Specifically, it is shown that there exists a threshold for the number of bit-pipes in the feedback link of transmitter-receiver pair 1 (resp. 2), denoted by $\overleftarrow{n}_{11}^{*}\left(\right.$ resp. $\left.\overleftarrow{n}_{22}^{*}\right)$ for which any $\overleftarrow{n}_{11}>\overleftarrow{n}_{11}^{*}\left(\right.$ resp. $\left.\overleftarrow{n}_{22}>\overleftarrow{n}_{22}^{*}\right)$ enlarges the capacity region, i.e., $\mathcal{C}\left(\vec{n}_{11}, \vec{n}_{22}, n_{12}, n_{21}, 0,0\right) \subset \mathcal{C}\left(\vec{n}_{11}, \vec{n}_{22}, n_{12}, n_{21}, \overleftarrow{n}_{11}, 0\right)$ (resp. $\left.\mathcal{C}\left(\vec{n}_{11}, \vec{n}_{22}, n_{12}, n_{21}, 0,0\right) \subset \mathcal{C}\left(\vec{n}_{11}, \vec{n}_{22}, n_{12}, n_{21}, 0, \overleftarrow{n}_{22}\right)\right)$. The exact conditions on $\overleftarrow{n}_{11}$ (resp. $\overleftarrow{n}_{22}$ ) to observe an improvement on a single rate or the sum-rate capacity, for any 4 -tuple $\left(\vec{n}_{11}, \vec{n}_{22}, n_{12}, n_{21}\right)$ $\in \mathbb{N}^{4}$ are also presented in this paper.
\end{abstract}

Key words: linear deterministic interference channel, noisy channel-output feedback, capacity region.

\footnotetext{
* The CITI Laboratory is a joint lab between the Institut National de Recherche en Informatique et en Automatique (INRIA), Université de Lyon and Institut National de Sciences Apliquées (INSA) de Lyon. This research was supported in part by the European Commission under Marie Sklodowska-Curie Individual Fellowship No. 659316 (CYBERNETS), Universidad del Cauca, Popayán, Colombia, and the Administrative Department of Science, Technology and Innovation of Colombia (Colciencias), fellowship No. 617-2013.
} 


\section{Introduction}

Channel-output feedback is an interference management technique that significantly increases the number of degrees of freedom (DoF) for the two-user Gaussian interference channel (IC) in most of the interference regimes 1 . Essentially, in the very strong interference regime, the DoF gain provided by perfectchannel output feedback can be arbitrarily large when the interference to noise ratios (INRs) and signal to noise ratios (SNRs) grow to infinity. One of the reasons why feedback provides such a surprising benefit stems from the fact that it uses interference to create alternative paths to the existing point-to-point paths. For instance, in the two-user IC, feedback creates a path from transmitter 1 (resp. transmitter 2) to receiver 1 (resp. receiver 2) in which symbols that are received at receiver 2 (resp. receiver 1 ) are fed back to transmitter 2 (resp. transmitter 1), which decodes the messages and retransmits them to receiver 1 (resp. receiver 2). Another metric to determine the benefits of feedback is the number of generalized DoF (GDoF), see 3 for a discussion on DoF and GDoF, as well as other metrics. The GDoF gain due to feedback in the IC depends on the topology of the network and the number of transmitter-receiver pairs in the network. For instance, in the symmetric $K$-user cyclic $Z$-interference channel, the DoF gain does not increase with $K$ 4. In particular, in the very strong interference regime, the DoF gain is shown to be monotonically decreasing with $K$. Alternatively, in the fully connected symmetric $K$-user IC with perfect feedback, the number of GDoF per user is shown to be identical to the one in the two-user case, with an exception in a particular singularity, and totally independent of the exact number of transmitter-receiver pairs 5. It is important to highlight that the network topology, the number of transmitter-receiver pairs and the interference regime are not the only parameters determining the effect of feedback. Indeed, the presence of noise in the feedback links turns out to be another relevant factor. As shown later in this paper, in the case in which one transmitter-receiver pair is in a high interference regime (the interfering signal is stronger than the intended signal) and the other is in a low interference regime (the interfering signal is weaker than the intended signal), the use of feedback in the former does not enlarge the capacity region, even in the case of perfect output feedback. Conversely, using feedback in the latter might enlarge the capacity region depending on the SNR of the feedback link. The exact values of the feedback SNRs beyond which the capacity region is enlarged depend on all the other channel parameters: two forward SNRs and two forward INRs. In [6], the capacity region of the two-user Gaussian IC (GIC) with noisy channel output feedback is approximated to within a constant number of bits for the symmetric case. These results are generalized in 11 for non-symmetric cases. However, from the available descriptions of the capacity regions with and without feedback, identifying whether or not the existence of a feedback link with a given SNR enlarges the capacity region is not a trivial task.

An alternative for dealing with the challenges described above is to study the GIC via its linear deterministic IC (LD-IC) approximation [7, for which the capacity region is perfectly known 8, 10. The two-user LD-IC with noisy channel output feedback (LD-IC-NOF) is fully described by six parameters: $\left(\vec{n}_{11}, \vec{n}_{22}, n_{12}, n_{21}, \overleftarrow{n}_{11}, \overleftarrow{n}_{22}\right) \in \mathbb{N}^{6}$. There exists a mapping between the parameters describing the two-user LD-IC and the parameters describing the GIC.

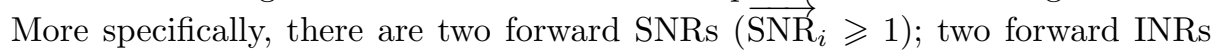


$\left(\operatorname{INR}_{i j} \geqslant 1\right)$; and two backward SNRs $\left(\overleftarrow{\mathrm{SNR}}_{i} \geqslant 1\right)$, with $i \in\{1,2\}$ and $j \in\{1,2\} \backslash\{i\}$. In the LD-IC, the parameters of the GIC are mapped into the number of bit-pipes between each transmitter and its corresponding intended receiver, i.e., $\vec{n}_{i i}=\left\lfloor\frac{1}{2} \log _{2}\left(\overrightarrow{\mathrm{SNR}}_{i}\right)\right\rfloor$; between transmitter $j$ and receiver $i$ i.e., $n_{i j}=\left\lfloor\frac{1}{2} \log _{2}\left(\mathrm{INR}_{i j}\right)\right\rfloor$; and between each receiver and its corresponding transmitter, i.e., $\overleftarrow{n}_{i i}=\left\lfloor\frac{1}{2} \log _{2}\left(\overleftarrow{\mathrm{SNR}}_{i}\right)\right\rfloor$. An LD-IC without feedback corresponds to the case in which $\overleftarrow{n}_{11}=\overleftarrow{n}_{22}=0$ and the capacity region is denoted by $\mathcal{C}\left(\vec{n}_{11}, \vec{n}_{22}, n_{12}, n_{21}, 0,0\right)$. In the case in which feedback is available at both transmitters, $\overleftarrow{n}_{11}>0$ and $\overleftarrow{n}_{22}>0$, the capacity is denoted by $\mathcal{C}\left(\vec{n}_{11}, \vec{n}_{22}, n_{12}, n_{21}, \overleftarrow{n}_{11}, \overleftarrow{n}_{22}\right)$

This paper presents the exact conditions on $\overleftarrow{n}_{11}$ (resp. $\overleftarrow{n}_{22}$ ) for observing an improvement in the capacity region $\mathcal{C}\left(\vec{n}_{11}, \vec{n}_{22}, n_{12}, n_{21}, \overleftarrow{n}_{11}, 0\right)$ (resp. $\left.\mathcal{C}\left(\vec{n}_{11}, \vec{n}_{22}, n_{12}, n_{21}, 0, \overleftarrow{n}_{22}\right)\right)$ with respect to $\mathcal{C}\left(\vec{n}_{11}, \vec{n}_{22}, n_{12}, n_{21}, 0,0\right)$, for any 4-tuple $\left(\vec{n}_{11}, \vec{n}_{22}, n_{12}, n_{21}\right) \in \mathbb{N}^{4}$. More specifically, it is shown that there exists a threshold for the number of bit-pipes in the feedback link of transmitterreceiver pair 1 (resp. 2), beyond which the capacity region of the two-user LDIC-NOF can be enlarged, i.e., $\mathcal{C}\left(\vec{n}_{11}, \vec{n}_{22}, n_{12}, n_{21}, 0,0\right) \subset \mathcal{C}\left(\vec{n}_{11}, \vec{n}_{22}, n_{12}\right.$, $\left.n_{21}, \overleftarrow{n}_{11}, 0\right)\left(\operatorname{resp} . \mathcal{C}\left(\vec{n}_{11}, \vec{n}_{22}, n_{12}, n_{21}, 0,0\right) \subset \mathcal{C}\left(\vec{n}_{11}, \vec{n}_{22}, n_{12}, n_{21}, 0, \overleftarrow{n}_{22}\right)\right)$ with strict inclusion. The exact conditions on $\overleftarrow{n}_{11}$ (resp. $\overleftarrow{n}_{22}$ ) to observe an improvement on a single rate or the sum-rate capacity, for any 4-tuple $\left(\vec{n}_{11}, \vec{n}_{22}, n_{12}, n_{21}\right) \in \mathbb{N}^{4}$ are also presented in this paper. Surprisingly, these values can be expressed in closed-form using relatively simple expressions that depend on some of the parameters $\vec{n}_{11}, \vec{n}_{22}, n_{12}$ and $n_{21}$.

Based on these results, several relevant engineering questions arise in this setting. For instance, in which of the two transmitter-receiver pairs must the feedback link be implemented if the objective is to improve: (a) the individual rate of the transmitter-receiver pair in which feedback is implemented; (b) the individual rate of the other transmitter-receiver pair; or (c) the sum-rate of both transmitter-receiver pairs. In each of these scenarios, the feedback SNR, either $\overleftarrow{n}_{11}$ or $\overleftarrow{n}_{22}$, must be bigger than a given threshold for the improvement to be observed. Interestingly, the answer is completely different for each of these scenarios. In addition to the results described above, the exact values of $\overleftarrow{n}_{11}$ or $\overleftarrow{n}_{22}$ for which feedback does not enlarge the capacity region are also identified.

\section{Linear Deterministic Interference Channel with Noisy-Channel Output Feedback}

Consider the two-user LD-IC-NOF, with parameters $\vec{n}_{11}, \vec{n}_{22}, n_{12}, n_{21}, \overleftarrow{n}_{11}$ and $\overleftarrow{n}_{22}$ described in Fig. 1. The parameters $\vec{n}_{i i}, n_{i j}$ and $\overleftarrow{n}_{i i}$ with $i \in\{1,2\}$ and $j \in\{1,2\} \backslash\{i\}$, are non-negative integers. Parameter $\vec{n}_{i i}$ represents the number of bit-pipes between transmitter $i$ and receiver $i$; parameter $n_{i j}$ represents the number of bit-pipes between transmitter $j$ and receiver $i$; and parameter $\overleftarrow{n}_{i i}$ represents the number of bit-pipes between receiver $i$ and transmitter $i$ (feedback). At transmitter $i$, with $i \in\{1,2\}$, the channel-input $\boldsymbol{X}_{i}^{(n)}$ during channel use $n$, with $n \in\{1, \ldots, N\}$, is a $q$-dimensional binary vector $\boldsymbol{X}_{i}^{(n)}=\left(X_{i, 1}^{(n)}, \ldots, X_{i, q}^{(n)}\right)^{\top}$, with

$$
q=\max \left(\vec{n}_{11}, \vec{n}_{22}, n_{12}, n_{21}\right)
$$




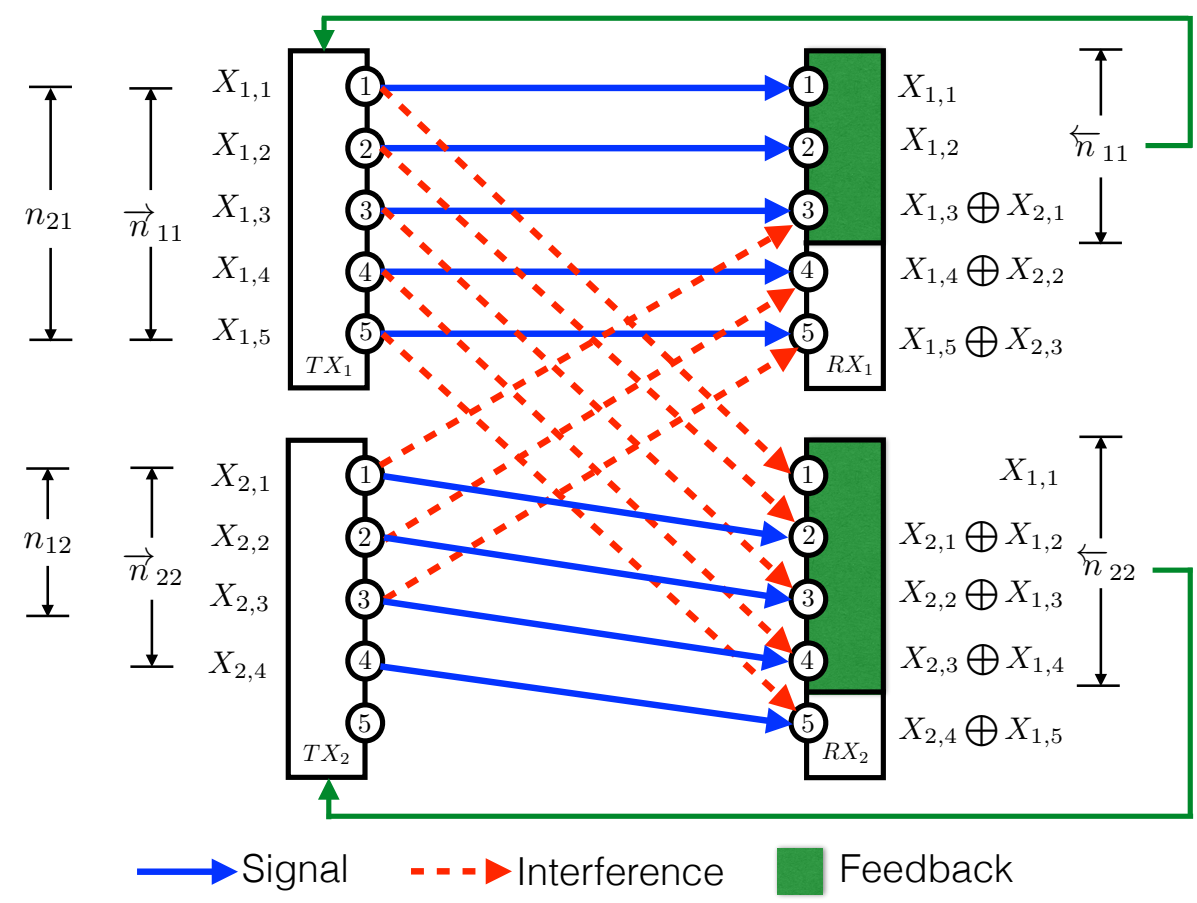

Fig. 1. Two-user linear deterministic interference channel with noisy channel-output feedback. The bit-pipe line number 1 represents the most significant bit.

and $N$ the block length. At receiver $i$, the channel-output $\overrightarrow{\boldsymbol{Y}}_{i}^{(n)}$ during channel use $n$ is also a $q$-dimensional binary vector $\overrightarrow{\boldsymbol{Y}}_{i}^{(n)}=\left(\vec{Y}_{i, 1}^{(n)}, \ldots, \vec{Y}_{i, q}^{(n)}\right)^{\top}$. Let $\boldsymbol{S}$ be a $q \times q$ lower shift matrix. The input-output relation during channel use $n$ is given as follows

$$
\overrightarrow{\boldsymbol{Y}}_{i}^{(n)}=\boldsymbol{S}^{q-\vec{n}_{i i}} \boldsymbol{X}_{i}^{(n)}+\boldsymbol{S}^{q-n_{i j}} \boldsymbol{X}_{j}^{(n)},
$$

and the feedback signal $\overleftarrow{\boldsymbol{Y}}_{i, n}$ available at transmitter $i$ at the end of channel use $n$ satisfies

$$
\left((0, \ldots, 0),\left(\overleftarrow{\boldsymbol{Y}}_{i}^{(n)}\right)^{\top}\right)^{\top}=\boldsymbol{S}^{\left(\max \left(\vec{n}_{i i}, n_{i j}\right)-\overleftarrow{n}_{i i}\right)^{+}} \overrightarrow{\boldsymbol{Y}}_{i}^{(n-d)}
$$

where $d$ is a finite feedback delay, additions and multiplications are defined over the binary field, and $(\cdot)^{+}$is the positive part operator. The dimension of the vector $(0, \ldots, 0)$ in (3) is $q-\min \left(\overleftarrow{n}_{i i}, \max \left(\vec{n}_{i i}, n_{i j}\right)\right)$ and the vector $\overleftarrow{\boldsymbol{Y}}_{i}^{(n)}$ represents the $\min \left(\overleftarrow{n}_{i i}, \max \left(\vec{n}_{i i}, n_{i j}\right)\right)$ least significant bits of $\boldsymbol{S}^{\left(\max \left(\vec{n}_{i i}, n_{i j}\right)-\overleftarrow{n}_{i i}\right)^{+}} \overrightarrow{\boldsymbol{Y}}_{i}^{(n-d)}$.

Transmitter $i$ sends $M_{i}$ information bits $b_{i, 1}, \ldots, b_{i, M_{i}}$ by sending the codeword $\left(\boldsymbol{X}_{i}^{(1)}, \ldots, \boldsymbol{X}_{i}^{(N)}\right)$. The encoder of transmitter $i$ can be modeled as a set 
of deterministic mappings $f_{i}^{(1)}, \ldots, f_{i}^{(N)}$, with $f_{i}^{(1)}:\{0,1\}^{M_{i}} \rightarrow\{0,1\}^{q}$ and $\forall n \in\{2, \ldots, N\}, f_{i}^{(n)}:\{0,1\}^{M_{i}} \times\{0,1\}^{q(n-1)} \rightarrow\{0,1\}^{q}$, such that

$$
\begin{aligned}
& \boldsymbol{X}_{i}^{(1)}=f_{i}^{(1)}\left(b_{i, 1}, \ldots, b_{i, M_{i}}\right) \text { and } \\
& \boldsymbol{X}_{i}^{(n)}=f_{i}^{(n)}\left(b_{i, 1}, \ldots, b_{i, M_{i}}, \overleftarrow{\boldsymbol{Y}}_{i}^{(1)}, \ldots, \overleftarrow{\boldsymbol{Y}}_{i}^{(n-1)}\right)
\end{aligned}
$$

At the end of the block, receiver $i$ uses the sequence $\boldsymbol{Y}_{i}^{(1)}, \ldots, \boldsymbol{Y}_{i}^{(N)}$ to generate the estimates $\hat{b}_{i, 1}, \ldots, \hat{b}_{i, M_{i}}$. The average bit error probability at receiver $i$, denoted by $p_{i}$, is calculated as follows

$$
p_{i}=\frac{1}{M_{i}} \sum_{\ell=1}^{M_{i}} \mathbb{1}_{\left\{\hat{b}_{i, \ell} \neq b_{i, \ell}\right\}} .
$$

A rate pair $\left(R_{1}, R_{2}\right) \in \mathbb{R}_{+}^{2}$ is said to be achievable if it satisfies the following definition.

Definition 1 (Achievable Rate Pairs). The rate pair $\left(R_{1}, R_{2}\right) \in \mathbb{R}_{+}^{2}$ is achievable if there exists at least one pair of codebooks $\mathcal{X}_{1}^{N}$ and $\mathcal{X}_{2}^{N}$ with codewords of length $N$, and the corresponding encoding functions $f_{1}^{(1)}, \ldots, f_{1}^{(N)}$ and $f_{2}^{(1)}, \ldots, f_{2}^{(N)}$ such that the average bit error probability can be made arbitrarily small by letting the block length $N$ grow to infinity.

The information capacity region is the closure of all information achievable rate pairs $\left(R_{1}, R_{2}\right)$. Denote by $\mathcal{C}\left(\vec{n}_{11}, \vec{n}_{22}, n_{12}, n_{21}, \overleftarrow{n}_{11}, \overleftarrow{n}_{22}\right)$ the capacity region of the two-user LD-IC-NOF with parameters $\vec{n}_{11}, \vec{n}_{22}, n_{12}, n_{21}, \overleftarrow{n}_{11}$, and $\bar{n}_{22}$. Lemma 1 fully characterizes the set $\mathcal{C}\left(\vec{n}_{11}, \vec{n}_{22}, n_{12}, n_{21}, \bar{n}_{11}, \overleftarrow{n}_{22}\right)$.

Lemma 1 (Lemma 6 in [10]). The capacity region $\mathcal{C}\left(\vec{n}_{11}, \vec{n}_{22}, n_{12}, n_{21}\right.$, $\left.\overleftarrow{n}_{11}, \overleftarrow{n}_{22}\right)$ of the two-user LD-IC-NOF is the set of non-negative rate pairs $\left(R_{1}, R_{2}\right)$ that satisfy $\forall i \in\{1,2\}$ and $j \in\{1,2\} \backslash\{i\}$ :

$$
\begin{aligned}
R_{i} \leqslant & \min \left(\max \left(\vec{n}_{i i}, n_{j i}\right), \max \left(\vec{n}_{i i}, n_{i j}\right)\right), \\
R_{i} \leqslant & \min \left(\max \left(\vec{n}_{i i}, n_{j i}\right), \max \left(\vec{n}_{i i}, \overleftarrow{n}_{j j}-\left(\vec{n}_{j j}-n_{j i}\right)^{+}\right)\right), \\
R_{1}+ & R_{2} \leqslant \min \left(\max \left(\vec{n}_{22}, n_{12}\right)+\left(\vec{n}_{11}-n_{12}\right)^{+}, \max \left(\vec{n}_{11}, n_{21}\right)\right. \\
& \left.+\left(\vec{n}_{22}-n_{21}\right)^{+}\right), \\
R_{1}+ & R_{2} \leqslant \max \left(\left(\vec{n}_{11}-n_{12}\right)^{+}, n_{21}, \vec{n}_{11}-\left(\max \left(\vec{n}_{11}, n_{12}\right)-\overleftarrow{n}_{11}\right)^{+}\right) \\
& +\max \left(\left(\vec{n}_{22}-n_{21}\right)^{+}, n_{12}, \vec{n}_{22}-\left(\max \left(\vec{n}_{22}, n_{21}\right)-\overleftarrow{n}_{22}\right)^{+}\right), \\
2 R_{i}+ & R_{j} \leqslant \max \left(\vec{n}_{i i}, n_{j i}\right)+\left(\vec{n}_{i i}-n_{i j}\right)^{+} \\
& +\max \left(\left(\vec{n}_{j j}-n_{j i}\right)^{+}, n_{i j}, \vec{n}_{j j}-\left(\max \left(\vec{n}_{j j}, n_{j i}\right)-\overleftarrow{n}_{j j}\right)^{+}\right) .
\end{aligned}
$$




\section{Preliminaries}

\subsection{Definitions}

Let $\alpha_{i} \in \mathbb{Q}$ be the interference regime of transmitter-receiver pair $i$, with $i \in$ $\{1,2\}$ and $j \in\{1,2\} \backslash\{i\}$,

$$
\alpha_{i}=\frac{n_{i j}}{\vec{n}_{i i}} .
$$

For each transmitter-receiver pair $i$, there exist five possible interference regimes (IRs): very weak IR (VWIR), i.e., $\alpha_{i} \leqslant \frac{1}{2}$, weak IR (WIR), i.e., $\frac{1}{2}<\alpha_{i} \leqslant \frac{2}{3}$, moderate IR (MIR), i.e., $\frac{2}{3}<\alpha_{i}<1$, strong IR (SIR), i.e., $1 \leqslant \alpha_{i} \leqslant 2$ and very strong IR (VSIR), i.e., $\alpha_{i}>2$ 9. The scenarios in which the desired signal is stronger than the interference $\left(\alpha_{i}<1\right)$, namely VWIR, WIR and MIR, are referred to as low-interference regimes (LIRs). Conversely, the scenarios in which the desired signal is weaker than or equal to the interference $\left(\alpha_{i} \geqslant 1\right)$, namely SIR and VSIR, are referred to as high-interference regimes (HIRs). In the twouser LD-IC, up to twenty-five possible interference regimes might emerge, given $\alpha_{1}$ and $\alpha_{2}$. However, only eight cases are of real interest. This is because the transmitter-receiver pairs can be indifferently labeled and thus, for instance, studying the case in which $\alpha_{1} \leqslant \frac{1}{2}$ and $\alpha_{2}>2$ is the same as studying the case in which $\alpha_{1}>2$ and $\alpha_{2} \leqslant \frac{1}{2}$.

The main results of this paper are presented using a list of events (Boolean variables) that are fully determined by the parameters $\vec{n}_{11}, \vec{n}_{22}, n_{12}$, and $n_{21}$. For instance, given the parameters $\left(\vec{n}_{11}, \vec{n}_{22}, n_{12}, n_{21}\right)$, the events $(9)$-(??) describe some combinations of interference regimes that are particularly interesting. Let $i \in\{1,2\}$ and $j \in\{1,2\} \backslash\{i\}$ and define the following events:

$$
\begin{array}{lr}
E_{1}: & \alpha_{1}<1 \wedge \quad \alpha_{2}<1,(i \text { and } j \text { in LIR }) \\
E_{2, i}: & \alpha_{i} \leqslant \frac{1}{2} \wedge 1 \leqslant \alpha_{j} \leqslant 2,(i \text { in VWIR and } j \text { in SIR }) \\
E_{3, i}: & \alpha_{i} \leqslant \frac{1}{2} \wedge \quad \alpha_{j}>2,(i \text { in VWIR and } j \text { in VSIR }) \\
E_{4, i}: & \frac{1}{2}<\alpha_{i} \leqslant \frac{2}{3} \wedge \quad \alpha_{j} \geqslant 1,(i \text { in WIR and } j \text { in HIR }) \\
E_{5, i}: & \frac{2}{3}<\alpha_{i}<1 \wedge \quad \alpha_{j} \geqslant 1,(i \text { in MIR and } j \text { in HIR }) \\
E_{6, i}: & \frac{1}{2}<\alpha_{i} \leqslant 1 \wedge \quad \alpha_{j}>1,(i \text { in WIR or MIR and } j \text { in HIR }) \\
E_{7, i}: & \alpha_{i} \geqslant 1 \wedge \quad \alpha_{j} \leqslant 1,(i \text { in HIR and } j \text { in LIR }) .
\end{array}
$$

Note that the event $E_{6, i}$ includes $\alpha_{i}=1$ and excludes $\alpha_{j}=1$, and the event $E_{7, i}$ includes $\alpha_{j}=1$.

Some other auxiliary events are considered. The event in which the signal from transmitter $i$ is stronger in its intended receiver than in its non-intended receiver is denoted by $E_{8, i}$, i.e.,

$$
E_{8, i}: \quad \vec{n}_{i i}>n_{j i} \text {. }
$$

The event in which the sum of the number of bit-pipes in the direct links is bigger than the sum of the number of bit-pipes in the cross-interference links is denoted by $E_{9}$, i.e., 


$$
E_{9}: \quad \vec{n}_{11}+\vec{n}_{22}>n_{12}+n_{21} .
$$

The event in which the sum of the number of bit-pipes in the direct links is bigger than the sum of the number of bit-pipes in one cross-interference link and twice the number of the bit-pipes in the other cross-interference link is denoted by $E_{10, i}$, i.e.,

$$
E_{10, i}: \quad \vec{n}_{i i}+\vec{n}_{j j}>n_{i j}+2 n_{j i} .
$$

Finally, the event in which the sum of the number of bit-pipes in the direct links is bigger than the number of bit-pipes in one cross-interference link is denoted by $E_{11, i}$, i.e.,

$$
E_{11, i}: \quad \vec{n}_{i i}+\vec{n}_{j j}<n_{i j}
$$

In the following, given an event, e.g. $E_{8, i}: \vec{n}_{i i}>n_{j i}$, the notation $\widetilde{E}_{8, i}$ implies $\vec{n}_{i i}<n_{j i} ; \bar{E}_{8, i}$ implies $\vec{n}_{i i} \leqslant n_{j i} ;$ and $\check{E}_{8, i}$ implies $\vec{n}_{i i} \geqslant n_{j i}$.

Combining the events (9)-(19), five main events are identified:

$$
\begin{aligned}
S_{1, i}: & \left(E_{1} \wedge E_{8, i}\right) \vee\left(E_{2, i} \wedge E_{8, i}\right) \vee\left(E_{3, i} \wedge E_{8, i} \wedge E_{9}\right) \vee\left(E_{4, i} \wedge E_{8, i} \wedge E_{9}\right) \vee\left(E_{5, i} \wedge E_{8, i} \wedge E_{9}\right),(20) \\
S_{2, i}: & \left(E_{3, i} \wedge \widetilde{E}_{8, j} \wedge \bar{E}_{9}\right) \vee\left(E_{6, i} \wedge \widetilde{E}_{8, j} \wedge \bar{E}_{9}\right) \vee\left(\widetilde{E}_{1} \wedge \widetilde{E}_{8, j}\right) \\
S_{3, i}: & \left(E_{1} \wedge \bar{E}_{8, i}\right) \vee\left(E_{2, i} \wedge \bar{E}_{8, i}\right) \vee\left(E_{3, i} \wedge \check{E}_{8, j} \wedge \bar{E}_{8, i}\right) \vee\left(E_{4, i} \wedge \check{E}_{8, j} \wedge \bar{E}_{8, i}\right) \\
& \vee\left(E_{5, i} \wedge \check{E}_{8, j} \wedge \bar{E}_{8, i}\right) \vee\left(\bar{E}_{1} \wedge \check{E}_{8, j}\right) \vee\left(E_{7, i}\right) \\
S_{4}: & E_{1} \wedge E_{8,1} \wedge E_{8,2} \wedge E_{10,1} \wedge E_{10,2} \\
S_{5}: & E_{1} \wedge E_{11,1} \wedge E_{11,2}
\end{aligned}
$$

For all $i \in\{1,2\}$ the events $S_{1, i}, S_{2, i}$ and $S_{3, i}$ exhibit the property stated by the following lemma.

Lemma 2. For all $i \in\{1,2\}$ and for all $\left(\vec{n}_{11}, \vec{n}_{22}, n_{12}, n_{21}\right) \in \mathbb{N}^{4}$, only one of the events among $S_{1, i}, S_{2, i}$ and $S_{3, i}$ is true.

Proof: The proof follows from verifying that for all $i \in\{1,2\}$ and $j \in$ $\{1,2\} \backslash\{i\}$, the events $20-(22)$ are mutually exclusive. For instance, consider that the event $\left(E_{1} \wedge E_{13, i}\right)$ in (20) is true. Then, $S_{1, i}$ is true and $E_{2, i}, E_{3, i}, E_{4, i}$, $E_{5, i}, E_{6, i}, E_{7, i}$ and $E_{8, i}$ hold false, which implies that $S_{2, i}$ and $S_{3, i}$ hold false as well, since all events in (21) and (22) are false. The same verification can be made for all the remaining events in (20). This proves that if $S_{1, i}$ is true then $S_{2, i}$ and $S_{3, i}$ hold simultaneously false. The same verification can be done for showing that when $S_{2, i}$ holds true (resp. $S_{3, i}$ ), both events $S_{1, i}$ and $S_{3, i}$ (resp. $S_{1, i}$ and $S_{2, i}$ ) hold simultaneously false. Finally following the same reasoning it can be verified that if any pair of the events $\left\{S_{1, i}, S_{2, i}, S_{3, i}\right\}$ is false, the remaining event is necessarily true. This completes the proof.

The events $S_{4}$ and $S_{5}$ exhibit the property stated by the following lemma.

Lemma 3. if one of the events $S_{4}$ or $S_{5}$ holds true, then the other necessarily holds false.

Proof: The proof of Lemma 3 follows along the same lines of the proof of Lemma 2 


\subsection{Rate Improvement Metrics}

The rate improvements are given in terms of the following metrics [8, 10]: (a) maximum individual rate improvements $\Delta_{1}$ and $\Delta_{2}$; and (b) maximum sum-rate improvement $\Sigma$, with $\Delta_{i} \in \mathbb{R}_{+}$and $\Sigma \in \mathbb{R}_{+}$for $i \in\{1,2\}$.

Let $\mathcal{C}_{1}=\mathcal{C}\left(\vec{n}_{11}, \vec{n}_{22}, n_{12}, n_{21}, \overleftarrow{n}_{11}, \overleftarrow{n}_{22}\right)$ and $\mathcal{C}_{2}=\mathcal{C}\left(\dot{\vec{n}}_{11}, \vec{n}_{22}, n_{12}, n_{21}, 0,0\right)$ be the capacity region with noisy channel-output feedback and without feedback, respectively. In order to formally define $\Delta_{1}, \Delta_{2}$ and $\Sigma$, consider a two-user LDIC-NOF with parameters $\vec{n}_{11}, \vec{n}_{22}, n_{12}, n_{21}, \overleftarrow{n}_{11}$, and $\overleftarrow{n}_{22}$. The maximum improvement of the individual rate $R_{i}, \Delta_{i}\left(\vec{n}_{11}, \vec{n}_{22}, n_{12}, n_{21}, \overleftarrow{n}_{11}, \overleftarrow{n}_{22}\right)$, due to the effect of channel-output feedback with respect to the case without feedback is

$$
\Delta_{i}\left(\vec{n}_{11}, \vec{n}_{22}, n_{12}, n_{21}, \overleftarrow{n}_{11}, \overleftarrow{n}_{22}\right)=\max _{R_{j}>0} \sup _{\left(R_{i}, R_{j}\right) \in \mathcal{C}_{1}} R_{i}-\sup _{\left(R_{i}^{\dagger}, R_{j}\right) \in \mathcal{C}_{2}} R_{i}^{\dagger}
$$

and the maximum improvement of the sum rate $\Sigma\left(\vec{n}_{11}, \vec{n}_{22}, n_{12}, n_{21}, \overleftarrow{n}_{11}, \overleftarrow{n}_{22}\right)$ with respect to the case without feedback is

$$
\Sigma\left(\vec{n}_{11}, \vec{n}_{22}, n_{12}, n_{21}, \overleftarrow{n}_{11}, \overleftarrow{n}_{22}\right)=\sup _{\left(R_{1}, R_{2}\right) \in \mathcal{C}_{1}} R_{1}+R_{2}-\sup _{\left(R_{1}^{\dagger}, R_{2}^{\dagger}\right) \in \mathcal{C}_{2}} R_{1}^{\dagger}+R_{2}^{\dagger}
$$

In the following, when feedback is exclusively used by transmitter-receiver pair $i$, i.e., $\overleftarrow{n}_{i i}>0$ and $\overleftarrow{n}_{j j}=0$, then the maximum improvement of the individual rate of transmitter-receiver $k$, with $k \in\{1,2\}$, and the maximum improvement of the sum rate are denoted by $\Delta_{k}\left(\vec{n}_{11}, \vec{n}_{22}, n_{12}, n_{21}, \overleftarrow{n}_{i i}\right)$ and $\Sigma\left(\vec{n}_{11}, \vec{n}_{22}, n_{12}, n_{21}, \overleftarrow{n}_{i i}\right)$, respectively. Hence, this notation $\Delta_{k}\left(\vec{n}_{11}, \vec{n}_{22}, n_{12}\right.$, $\left.n_{21}, \overleftarrow{n}_{i i}\right)$ replaces either $\Delta_{k}\left(\vec{n}_{11}, \vec{n}_{22}, n_{12}, n_{21}, \overleftarrow{n}_{11}, 0\right)$ or $\Delta_{k}\left(\vec{n}_{11}, \vec{n}_{22}, n_{12}\right.$, $n_{21}, 0, \bar{n}_{22}$ ), when $i=1$ or $i=2$, respectively. The same holds for the notation $\Sigma\left(\vec{n}_{11}, \vec{n}_{22}, n_{12}, n_{21}, \overleftarrow{n}_{i i}\right)$ that replaces $\Sigma\left(\vec{n}_{11}, \vec{n}_{22}, n_{12}, n_{21}, \overleftarrow{n}_{11}, 0\right)$ or $\Sigma\left(\vec{n}_{11}, \vec{n}_{22}, n_{12}, n_{21}, 0, \breve{n}_{22}\right)$, when $i=1$ or $i=2$, respectively.

\section{Main Results}

\subsection{Enlargement of the Capacity Region}

In this subsection, the capacity region of a two-user LD-IC-NOF with parameters $\left(\vec{n}_{11}, \vec{n}_{22}, n_{12}, n_{21}\right)$, when feedback is available only at transmitter-receiver pair $i$, i.e., $\overleftarrow{n}_{i i}>0$ and $\overleftarrow{n}_{j j}=0$, is denoted by $\mathcal{C}\left(\vec{n}_{11}, \vec{n}_{22}, n_{12}, n_{21}, \overleftarrow{n}_{i i}\right)$ instead of $\mathcal{C}\left(\vec{n}_{11}, \vec{n}_{22}, n_{12}, n_{21}, \overleftarrow{n}_{11}, 0\right)$ or $\mathcal{C}\left(\vec{n}_{11}, \vec{n}_{22}, n_{12}, n_{21}, 0, \overleftarrow{n}_{22}\right)$, when $i=1$ or $i=2$, respectively. Following this notation, Theorem 1 identifies the exact values of $\overleftarrow{n}_{i i}$ for which the strict inclusion $\mathcal{C}\left(\vec{n}_{11}, \vec{n}_{22}, n_{12}, n_{21}, 0,0\right) \subset$ $\mathcal{C}\left(\vec{n}_{11}, \vec{n}_{22}, n_{12}, n_{21}, \overleftarrow{n}_{i i}\right)$ holds, with $i \in\{1,2\}$.

Theorem 1. Let $i \in\{1,2\}, j \in\{1,2\} \backslash\{i\}$ and $\overleftarrow{n}_{i i}^{*} \in \mathbb{N}$ be

$$
\overleftarrow{n}_{i i}^{*}= \begin{cases}\max \left(n_{j i},\left(\vec{n}_{i i}-n_{i j}\right)^{+}\right) & \text {if } S_{1, i}=\text { True } \\ \vec{n}_{j j}+\left(\vec{n}_{i i}-n_{i j}\right)^{+} & \text {if } S_{2, i}=\text { True }\end{cases}
$$


Assume that $S_{3, i}=$ True. Then, for all $\overleftarrow{n}_{i i} \in \mathbb{N}, \mathcal{C}\left(\vec{n}_{11}, \vec{n}_{22}, n_{12}, n_{21}, 0,0\right)=$ $\mathcal{C}\left(\vec{n}_{11}, \vec{n}_{22}, n_{12}, n_{21}, \overleftarrow{n}_{i i}\right)$. Assume that either $S_{1, i}=$ True or $S_{2, i}=$ True. Then for all $\overleftarrow{n}_{i i} \leqslant \overleftarrow{n}_{i i}^{*}, \mathcal{C}\left(\vec{n}_{11}, \vec{n}_{22}, n_{12}, n_{21}, 0,0\right)=\mathcal{C}\left(\vec{n}_{11}, \vec{n}_{22}, n_{12}, n_{21}, \overleftarrow{n}_{i i}\right)$ and for all $\overleftarrow{n}_{i i}>\overleftarrow{n}_{i i}^{*}, \mathcal{C}\left(\vec{n}_{11}, \vec{n}_{22}, n_{12}, n_{21}, 0,0\right) \subset \mathcal{C}\left(\vec{n}_{11}, \vec{n}_{22}, n_{12}, n_{21}, \overleftarrow{n}_{i i}\right)$

Proof: The proof of Theorem 1 is presented in [12].

Theorem 1 shows that under event $S_{3, i}$ in $\sqrt{22}$, implementing feedback in transmitter-receiver pair $i$ does not bring any capacity region enlargement. Alternatively, under events $S_{1, i}$ in $(20)$ and $S_{2, i}$ in (21), the capacity region can be enlarged when $\overleftarrow{n}_{i i}>\overleftarrow{n}_{i i}^{*}$. That is, there exists a threshold on the SNR of the feedback link beyond which it is possible to observe a capacity region enlargement.

An interesting observation is that the threshold $\overleftarrow{n}_{i i}^{*}$ beyond which feedback is useful is different under event $S_{1, i}$ in 20 and event $S_{2, i}$ in (21). In general when $S_{1, i}$ holds true, the enlargement of the capacity region is due to the fact that feedback allows using interference as side information 13 . More specifically, when feedback is used at transmitter-receiver pair $i$ and $\overleftarrow{n}_{i i}>\max \left(n_{j i},\left(\vec{n}_{i i}-n_{i j}\right)^{+}\right)$, transmitter $i$ obtains part of the information sent by transmitter $j$. This information can be re-transmitted by transmitter $i$ to cancel the interference it produced at receiver $i$ when it was first transmitted by transmitter $j$. Interestingly, the interference perceived at receiver $j$ due to this re-transmission can be cancelled given that this information was reliably decoded when it was first sent by transmitter $j$. This allows transmitter-receiver pair $i$ or $j$ to improve its individual rate.

Alternatively, when $S_{2, i}$ in (21) holds true, the enlargement of the capacity region occurs thanks to the fact that some of the bits that cannot be transmitted directly from transmitter $j$ to receiver $j$, that is, those transmitted via the bitpipes $\vec{n}_{j j}+1, \ldots, \max \left(\vec{n}_{j j}, n_{i j}\right)$, can arrive to receiver $j$ via an alternative path: transmitter $j$ - receiver $i$ - transmitter $i$ - receiver $j$. For this to be possible at least the $\left(\vec{n}_{j j}+\left(\vec{n}_{i i}-n_{i j}\right)^{+}+1\right)$-th (feedback) bit-pipe from receiver $i$ to transmitter $i$ must be noise-free, i.e., $\overleftarrow{n}_{i i}>\vec{n}_{j j}+\left(\vec{n}_{i i}-n_{i j}\right)^{+}$.

\subsection{Improvement of the Individual Rate $R_{i}$ by Using Feedback in Link $i$}

Implementing channel output feedback in transmitter-receiver pair $i$ might allow increasing the individual rate of either transmitter-receiver pair $i$ or $j$. Theorem 2 identifies the exact values of $\overleftarrow{n}_{i i}$ for which the individual rate $R_{i}$ can be improved, given the parameters $\left(\vec{n}_{11}, \vec{n}_{22}, n_{12}, n_{21}\right)$ and $\overleftarrow{n}_{j j}=0$ in the twouser LD-IC-NOF.

Theorem 2. Let $i \in\{1,2\}, j \in\{1,2\} \backslash\{i\}$ and $\overleftarrow{n}_{i i}^{\dagger} \in \mathbb{N}$ be

$$
\overleftarrow{n}_{i i}^{\dagger}=\max \left(n_{j i},\left(\vec{n}_{i i}-n_{i j}\right)^{+}\right)
$$

Assume that either $S_{2, i}=$ True or $S_{3, i}=$ True. Then, for all $\overleftarrow{n}_{i i} \in \mathbb{N}, \Delta_{i}\left(\vec{n}_{11}\right.$, $\left.\vec{n}_{22}, n_{12}, n_{21}, \overleftarrow{n}_{i i}\right)=0$. Assume that $S_{1, i}=$ True. Then, when $\overleftarrow{n}_{i i} \leqslant \overleftarrow{n}_{i i}^{\dagger}$, it holds that $\Delta_{i}\left(\vec{n}_{11}, \vec{n}_{22}, n_{12}, n_{21}, \overleftarrow{n}_{i i}\right)=0$; and when $\overleftarrow{n}_{i i}>\overleftarrow{n}_{i i}^{\dagger}$, it holds that $\Delta_{i}\left(\vec{n}_{11}, \vec{n}_{22}, n_{12}, n_{21}, \overleftarrow{n}_{i i}\right)>0$. 
Proof: The proof of Theorem 2 is presented in 12 .

Theorem 2 highlights that under events $S_{2, i}$ in 21 and $S_{3, i}$ in 22 , the individual rate $R_{i}$ cannot be improved by using feedback in transmitter-receiver pair $i$, i.e., $\Delta_{i}\left(\vec{n}_{11}, \vec{n}_{22}, n_{12}, n_{21}, \overleftarrow{n}_{i i}\right)=0$. Alternatively, under event $S_{1, i}$ in $(20)$ the individual rate $R_{i}$ can be improved, i.e., $\Delta_{i}\left(\vec{n}_{11}, \vec{n}_{22}, n_{12}, n_{21}, \overleftarrow{n}_{i i}\right)>0$, when $\overleftarrow{n}_{i i}>\max \left(n_{j i},\left(\vec{n}_{i i}-n_{i j}\right)^{+}\right)$

It is worth noting that under event $S_{3, i}$ in 22 , the capacity region cannot be improved via feedback (Theorem 1) and thus, none of the individual rates can be improved as suggested by Theorem 2 Alternatively, under event $S_{2, i}$ in (21), the capacity region can be enlarged (Theorem 1) but the individual rate $R_{i}$ cannot be improved (Theorem 2). This implies that the capacity improvement occurs due to the fact that $R_{j}$ can be improved. More specifically, in this case: $\Delta_{i}\left(\vec{n}_{11}, \vec{n}_{22}, n_{12}, n_{21}, \overleftarrow{n}_{i i}\right)=0$ and $\Delta_{j}\left(\vec{n}_{11}, \vec{n}_{22}, n_{12}, n_{21}, \overleftarrow{n}_{i i}\right)>0$. This implies that using feedback in transmitter-receiver pair $i$ is exclusively beneficial for transmitter-receiver pair $j$, as shown in the following section.

\subsection{Improvement of the Individual Rate $R_{j}$ by Using Feedback in Link $i$}

Implementing channel output feedback in transmitter-receiver pair $i$ might allow increasing the individual rate of transmitter-receiver pair $i$ or $j$; or both individual rates. This reveals the altruistic nature of implementing feedback as suggested in [2]. Theorem 3 identifies the exact values of $\overleftarrow{n}_{i i}$ for which the individual rate $R_{j}$ can be improved by using feedback in transmitter-receiver pair $i$, given the parameters $\left(\vec{n}_{11}, \vec{n}_{22}, n_{12}, n_{21}\right)$ and $\overleftarrow{n}_{j j}=0$ in the two-user LD-IC-NOF.

Theorem 3. Let $i \in\{1,2\}, j \in\{1,2\} \backslash\{i\}$ and $\overleftarrow{n}_{i i}^{\ddagger} \in \mathbb{N}$ be

$$
\overleftarrow{n}_{i i}^{\ddagger}= \begin{cases}\max \left(n_{j i},\left(\vec{n}_{i i}-n_{i j}\right)^{+}\right) & \text {if } S_{1, i}=\text { True } \\ \vec{n}_{j j}+\left(\vec{n}_{i i}-n_{i j}\right)^{+} & \text {if } S_{2, i}=\text { True. }\end{cases}
$$

Assume that $S_{3, i}=$ True. Then, for all $\overleftarrow{n}_{i i} \in \mathbb{N}, \Delta_{j}\left(\vec{n}_{11}, \vec{n}_{22}, n_{12}, n_{21}, \overleftarrow{n}_{i i}\right)=$ 0 . Assume that either $S_{1, i}=$ True or $S_{2, i}=$ True. Then, when $\overleftarrow{n}_{i i} \leqslant \overleftarrow{n}_{i i}^{\ddagger}$, it holds that $\Delta_{j}\left(\vec{n}_{11}, \vec{n}_{22}, n_{12}, n_{21}, \overleftarrow{n}_{i i}\right)=0$; and when $\overleftarrow{n}_{i i}>\overleftarrow{n}_{i i}^{\ddagger}$, it holds that $\Delta_{j}\left(\vec{n}_{11}, \vec{n}_{22}, n_{12}, n_{21}, \overleftarrow{n}_{i i}\right)>0$

Proof: The proof of Theorem 3 is presented in [12].

Theorem 3 shows that under event $S_{3, i}$ in 22, implementing feedback in transmitter-receiver pair $i$ does not bring any improvement on the rate $R_{j}$. This is in line with the results of Theorem 1 that states that under event $S_{3, i}$ in (22), implementing feedback in transmitter-receiver pair $i$ does not enlarge the capacity region.

In contrast, under events $S_{1, i}$ in 20 and $S_{2, i}$ in 21), the individual rate $R_{j}$ can be improved $\left(\Delta_{j}\left(\vec{n}_{11}, \vec{n}_{22}, n_{12}, n_{21}, \overleftarrow{n}_{i i}\right)>0\right)$ when $\overleftarrow{n}_{i i}>\overleftarrow{n}_{i i}^{\ddagger}$. It is important to highlight that under event $S_{1, i}$, the threshold on $\overleftarrow{n}_{i i}$ for increasing the individual rate $R_{i}$ i.e., $\left(\overleftarrow{n}_{i i}^{\dagger}\right)$, and $R_{j}$ i.e., $\left(\overleftarrow{n}_{i i}^{\ddagger}\right)$, are identical, see Theorem 2 and Theorem 3 . This shows that in this case, the use of feedback in transmitterreceiver pair $i$, with $\overleftarrow{n}_{i i}>\overleftarrow{n}_{i i}^{\dagger}=\overleftarrow{n}_{i i}^{\ddagger}$, simultaneously improves both individual 
rates. Under event $S_{2, i}$, using feedback in transmitter-receiver pair $i$, with $\overleftarrow{n}_{i i}>$ $\overleftarrow{n}_{i i}^{\ddagger}$, exclusively benefits transmitter-receiver pair $j$, which can improve its own individual rate.

\subsection{Improvement of the Sum-Capacity}

Implementing channel output feedback in transmitter-receiver pair $i$ increases the sum-capacity under certain conditions. Theorem 4 identifies the exact values of $\overleftarrow{n}_{i i}$ for which the sum-capacity can be improved, for parameters $\left(\vec{n}_{11}, \vec{n}_{22}, n_{12}, n_{21}\right)$ and $\overleftarrow{n}_{j j}=0$ in the two-user LD-IC-NOF.

Theorem 4. Let $i \in\{1,2\}, j \in\{1,2\} \backslash\{i\}$ and $\overleftarrow{n}_{i i}^{+} \in \mathbb{N}$ be

$$
\overleftarrow{n}_{i i}^{+}= \begin{cases}\max \left(n_{j i},\left(\vec{n}_{i i}-n_{i j}\right)^{+}\right) & \text {if } S_{4}=\text { True } \\ \vec{n}_{j j}+\left(\vec{n}_{i i}-n_{i j}\right)^{+} & \text {if } S_{5}=\text { True }\end{cases}
$$

Assume that $S_{4}=$ False and $S_{5}=$ False. Then, for all $\overleftarrow{n}_{i i} \in \mathbb{N}, \Sigma\left(\vec{n}_{11}, \vec{n}_{22}, n_{12}\right.$, $\left.n_{21}, \overleftarrow{n}_{i i}\right)=0$. Assume that $S_{4}=$ True or $S_{5}=$ True. Then, when $\overleftarrow{n}_{i i} \leqslant \overleftarrow{n}_{i i}^{+}$, it holds that $\Sigma\left(\vec{n}_{11}, \vec{n}_{22}, n_{12}, n_{21}, \overleftarrow{n}_{i i}\right)=0$; and when $\overleftarrow{n}_{i i}>\overleftarrow{n}_{i i}^{+}$, it holds that $\Sigma\left(\vec{n}_{11}, \vec{n}_{22}, n_{12}, n_{21}, \overleftarrow{n}_{i i}\right)>0$.

Proof: The proof of Theorem 4 is presented in 12 .

Theorem 4 identifies the conditions under which implementing feedback in transmitter-receiver pair $i$ improves the sum-capacity whenever $\overleftarrow{n}_{i i}>\overleftarrow{n}_{i i}^{+}$, that is, $\Sigma\left(\vec{n}_{11}, \vec{n}_{22}, n_{12}, n_{21}, \overleftarrow{n}_{i i}\right)>0$. Theorem 4 highlights that one of the necessary but not sufficient conditions for improving the sum-capacity by implementing feedback in transmitter-receiver pair $i$ is that either $(a)$ both transmitterreceiver pairs must be in LIR; or $(b)$ both transmitter-receiver pairs must be in HIR. This follows immediately from observing that for $S_{4}$ or $S_{5}$ to hold true, the event $E_{1}$ or $\bar{E}_{1}$ must hold true.

Interestingly, Theorem 4 shows that if one transmitter-receiver pair is in LIR and the other transmitter-receiver pair is in HIR, then the sum-capacity cannot be improved. Finally, note that the thresholds $\overleftarrow{n}_{i i}^{+}$in the events $S_{4}$ and $S_{5}$ coincide with those observed in Theorem 1 .

\section{Conclusions}

This paper presented the exact conditions on the feedback parameters $\overleftarrow{n}_{11}$ and $\overleftarrow{n}_{22}$, beyond which the capacity region of the two-user LD-IC-NOF can be enlarged for any 4 -tuple $\left(\vec{n}_{11}, \vec{n}_{22}, n_{12}, n_{21}\right) \in \mathbb{N}^{4}$. More specifically, the exact values of $\overleftarrow{n}_{11}$ (resp. $\overleftarrow{n}_{22}$ ) for which $\mathcal{C}\left(\vec{n}_{11}, \vec{n}_{22}, n_{12}, n_{21}, 0,0\right) \subset \mathcal{C}\left(\vec{n}_{11}, \vec{n}_{22}, n_{12}\right.$, $\left.n_{21}, \overleftarrow{n}_{11}, 0\right)\left(\operatorname{resp} . \mathcal{C}\left(\vec{n}_{11}, \vec{n}_{22}, n_{12}, n_{21}, 0,0\right) \subset \mathcal{C}\left(\vec{n}_{11}, \vec{n}_{22}, n_{12}, n_{21}, 0, \overleftarrow{n}_{22}\right)\right)$ with strict inclusion. The exact conditions on $\overleftarrow{n}_{11}$ (resp. $\overleftarrow{n}_{22}$ ) to observe an improvement on a single rate or the sum-rate capacity, for any 4-tuple $\left(\vec{n}_{11}, \vec{n}_{22}, n_{12}, n_{21}\right) \in \mathbb{N}^{4}$ were also presented. Interestingly, there exist conditions in the two-user LD-IC-NOF in which the use of feedback does not enlarge the capacity region. 


\section{References}

1. Suh, C., Tse, D. N. C.: Feedback Capacity of the Gaussian Interference Channel to within 2 Bits. IEEE Transactions on Information Theory, vol. 57, no. 5, pp. 2667-2685. (2011).

2. Perlaza, S. M., Tandon, R., Poor, H. V., Han, Z.: Perfect Output Feedback in the Two-User Decentralized Interference Channel. IEEE Transactions on Information Theory, vol. 61, no. 10, pp.5441-5462. (2015).

3. Jafar, S. A.: Interference Alignment: A New Look at Signal Dimensions in a Communication Network. Foundations and Trends in Communications and Information Theory, vol. 7, no. 1 pp. 1-134. (2010).

4. Tandon, R., Mohajer, S. and Poor, H. V.: On the Symmetric Feedback Capacity of the K-User Cyclic Z-Interference Channel. IEEE Transactions on Information Theory, vol. 59, no. 5, pp. 2713-2734. (2013).

5. Mohajer, S., Tandon, R., Poor, H. V.: On the Feedback Capacity of the Fully Connected $K$-User Interference Channel. IEEE Transactions on Information Theory, vol. 59, no. 5, pp. 2863-2881. (2013).

6. Le, S-Q., Tandon, R., Motani, M., Poor, H. V.: Approximate Capacity Region for the Symmetric Gaussian Interference Channel with Noisy Feedback. IEEE Transactions on Information Theory, vol. 61, no. 7, pp. 3737-3762. (2015).

7. Avestimehr, S., Diggavi, S., Tse, D. N. C.: Wireless Network Information Flow: a Deterministic Approach. IEEE Transactions on Information Theory, vol. 57, no. 4, pp. 1872-1905. (2011).

8. Quintero, V., Perlaza, S. M. Gorce, J-M.: Noisy Channel-Output Feedback Capacity of the Linear Deterministic Interference Channel. In: IEEE Information Theory Workshop. Jeju Island, Korea. (2015).

9. Etkin, R. H., Tse, D. N. C., Hua, W.: Gaussian Interference Channel Capacity to within One Bit. IEEE Transactions on Information Theory, vol. 54, no. 12, pp. 5534-5562. (2008).

10. Quintero, V., Perlaza, S. M., Gorce, J.-M.: Noisy Channel-Output Feedback Capacity of the Linear Deterministic Interference Channel. INRIA Grenoble - RhôneAlpes, Lyon, France, Tech. Rep. 456, Jan. (2015).

11. Quintero, V., Perlaza, S. M., Esnaola, I., Gorce, J.-M.: Approximated Capacity of the Two-User Gaussian Interference Channel with Noisy Channel-Output Feedback. INRIA Grenoble - Rhône- Alpes, Lyon, France, Tech. Rep. 8861, Mar. (2016).

12. Quintero, V., Perlaza, S. M., Esnaola, I., Gorce, J.-M.: When Does Channel-Output Feedback Enlarge the Capacity Region of the Two-User Linear Deterministic Interference Channel?. INRIA Grenoble - Rhône- Alpes, Lyon, France, Tech. Rep. 8862, Mar. (2016).

13. Shannon C.E. Channels with side information at the transmitter. IBM Journal of Research and Development, vol. 2, no. 4, pp. 289-293. (1958). 